\title{
Politiques de population et réduction de la fécondité au Burkina Faso : limites et perspectives
}

\author{
Zan L. Moussa \\ Institut Supérieur des Sciences de la Population (ISSP), Université Ouaga I Pr. Ki-Zerbo, Burkina \\ Faso. \\ lonkilazan@yahoo.fr
}

\section{Résumé}

Cet article vise à comprendre l'action des politiques de population sur la pratique contraceptive et la fécondité selon le niveau d'instruction et le milieu de résidence au Burkina Faso. II utilise les données issues des Enquêtes démographiques et de santé de 1998 et 2010 du Burkina Faso ainsi que la méthode de la décomposition des sources du changement. II y ressort que les politiques de planification familiale ont eu d'importants effets sur la connaissance et la pratique contraceptive. Cependant, l'impact de la pratique contraceptive moderne sur la baisse de la fécondité reste assez limité, montrant ainsi l'importance des autres politiques de développement dans la baisse de la fécondité. Nous recommandons une poursuite des politiques de planification familiale mais l'Etat doit aussi mettre l'accent sur l'accès des filles et des femmes à l'éducation et l'amélioration des conditions de vie des populations.

Mots clés : Politique de population, Planification familiale, Contraception, Croissance démographique, Fécondité

\section{ABSTRACT}

This article aims to understand the action of population policies on contraceptive use and fertility according to the variation of level of education and residence area in Burkina Faso. It uses data from the Burkina Demographic and health survey of 1998 and 2010 and the "method of decomposition". It appears that family planning policies have had important effects on contraceptive knowledge and use. However, the impact of modern contraceptive use on fertility decline is quite limited thus showing the importance of other development policies in the fertility decline. We recommend a continuation of family planning policies but the country must be focusing on the access of girls and women to education and the improvement of living conditions of the population.

Keywords: Population policy, Family Planning, Contraception, Population Growth, Fertility

\section{Introduction}

Le Burkina Faso a longtemps été caractérisé par une forte croissance démographique qui semble constituer un obstacle à son développement. En effet, l'indice synthétique de fécondité (ISF) qui désigne le nombre moyen d'enfants par femme en fin de vie féconde, a été estimé à 6,1 en 1960, 6,7 en 1976, 7,2 en 1985 et 7,3 en 199I (INSD 1995). Cette hausse de I'ISF à induit une forte croissance démographique dans un contexte marqué par une faible croissance économique. Cela a conduit le pays à prendre des mesures visant la maîtrise de la croissance démographique. Parmi ces mesures on peut citer l'adoption et la mise en œuvre de la Politique nationale de population (PNP) de 199I qui a été révisée en 2000 . Cette politique a eu pour but, entre autres, d'atteindre l'équilibre entre l'accroissement de la population et le développement socio-économique. Ces efforts se sont concrétisés par une augmentation de la prévalence contraceptive moderne des femmes en union qui est passée de $4,83 \%$ en 1998 à $15 \%$ en 2010 . Au cours de la même période on a noté une légère baisse de la fécondité de 7 à 6 enfants par femme selon le rapport de l'Enquête démographique et de santé (EDS) de 2010 (INSD et ICF 20I2).

Malgré cette baisse de la fécondité, la population du Burkina Faso a connu une forte croissance. De 7 964705 en 1985, elle est passée à 15730977 habitants en 2010, soit un doublement en 25 ans. Pendant ce temps, on a observé une variation du taux 
d'accroissement démographique qui atteint un pic de 3, I entre 1996 et 2006 . Cela n'a permis ni d'endiguer la pauvreté (dont l'incidence a été estimé à environ $46 \%$ de la population en 1994 et en 2009) ni d'étendre l'offre de santé (en 2010, 83,1\% des formations sanitaires remplissaient les normes en personnel et on dénombre 2063 habitants par médecin) (MS 2014). Pourtant, l'accès difficile à la santé, notamment la santé de la reproduction est responsable d'une mortalité maternelle. Le niveau de mortalité maternelle est parmi les plus élevés au monde : 371 décès maternels pour 100000 naissances vivantes, contre 216 décès maternels pour 100000 naissances vivantes au niveau mondial (OMS 2015). Les besoins non satisfaits en matière de contraception restaient importants, concernant $23,8 \%$ des femmes en 2010 contre $29 \%$ en 2003. Selon des analyses basées sur les données de l'EDS 2010 du Burkina Faso, une grossesse sur trois n'est pas intentionnelle et une grossesse non intentionnelle sur trois se termine par un avortement (Bankole et al. 2013 : p4). Selon le Ministère de la Santé (2006), 30 $\%$ des cas de mortalité maternelle au Burkina pourraient être évités grâce à un renforcement de sa politique de Planification Familiale.

Un renouveau de l'engagement international en faveur de la Planification Familiale a été promulgué lors de la conférence de haut niveau sur le thème " Population, développement et planification familiale en Afrique de l'Ouest francophone : 'urgence d'agir " abritée par le Burkina Faso, du 8 au 10 février 201 I à Ouagadougou. Dans le cadre du partenariat de Ouagadougou, le Burkina Faso a entrepris un certain nombre d'initiatives majeures dont l'élaboration de son Plan national de relance de la Planification Familiale en juillet 20I2. Dans ce plan, le gouvernement a défini l'objectif d'une évolution volontariste de la prévalence contraceptive (I,5 points de pourcentage d'augmentation par an) qui permettrait de limiter la population du Burkina Faso à 39 millions d'habitants en 2050 (Burkina Faso 20I3). Ainsi, le contexte actuel du Burkina Faso est caractérisé par la mise en œuvre de plusieurs actions visant la baisse de la fécondité à travers la promotion de la planification familiale. Aussi, devons-nous nous interroger sur la contribution de la planification familiale dans la baisse de la fécondité au Burkina Faso. Si cette contribution semble logique à plusieurs égards, la mesure de son poids dans l'ensemble des facteurs de la fécondité l'est moins. En effet, comme nous le verrons plus bas, d'autres actions de développement qui ne visent pas directement la réduction de la fécondité pourraient $y$ avoir contribué. Cela a été noté par Anoh (2004, p.I) en ces termes : " la diminution de la fécondité dans les milieux instruits et urbanisés est en effet le plus 2602 souvent antérieure aux programmes de planification familiale ". Cet article se pose donc la question suivante : quelle est la contribution des politiques de population dans les changements en matière de contraception moderne et de fécondité entre 1998 et 2010 au Burkina Faso ? De manière spécifique, il s'agit d'estimer les contributions des politiques de planification dans les changements observés en matière de connaissance et d'utilisation des méthodes contraceptives modernes et en matière de baisse de la fécondité.

\section{REVUE DE LA LITTERATURE}

La reproduction démographique est fonction d'un ensemble de facteurs qui ont été diversement appréhendés par les chercheurs. On distingue six approches théoriques dans l'explication des transitions de la fécondité (Piché $V$ et Poirier J 1995). Ce sont : l'accès aux moyens de contraception ; la théorie des flux intergénérationnels de richesse ; les systèmes de production; les facteurs institutionnels ; les facteurs socioculturels et le courant féministe. La contraception est devenue le moyen de recours le plus important pour une population qui cherche à limiter les naissances dans un régime de fécondité contrôlée (Leridon 2002). Elle est actuellement le levier le plus important des politiques de population et de planification familiale même si, avant ces politiques, certaines méthodes contraceptives étaient utilisées sous des formes et pour des fins différentes.

Politique de planification familiale, contraception et fécondité

Les relations entre fécondité et contraception semblent dépendre des besoins de régulation de la fécondité tant au niveau individuel que collectif. Dans une recherche expérimentale sur $|4|$ villages de Matlab réalisée par Joshi et Schultz (2007), un programme avec un volet de planification familiale a été mis en place entre 1974 à 1996. Dans le cadre de ce programme, deux agents de santé communautaire faisaient des visites bihebdomadaires aux femmes mariées âgées de 15-49 ans et les encourageaient à adopter une méthode contraceptive. A l'issue de cette intervention, une baisse de fécondité de 15\% a été constatée en 1986 dans les villages d'intervention par rapport aux villages de contrôle. Cependant, dans d'autres contextes, les relations entre planification familiale, contraception et baisse de la fécondité ne sont pas linéaires. En effet, la littérature montre que les facteurs socioculturels ont aussi une grande influence sur les pratiques en matière de fécondité. Selon Locoh (1992, p.273) : " les sociétés africaines ont été "orientées" pendant des siècles vers la 
préservation d'une fécondité élevée pour assurer leur simple survie démographique. Les régimes matrimoniaux, le statut des femmes, la valorisation des descendances nombreuses, l'organisation des familles devaient, avec des options diverses bien entendu, contribuer à l'expansion démographique, toujours problématique ". Les systèmes et les stratégies de production peuvent aussi être à l'origine des comportements en matière de fécondité comme le prouve cet exemple issu du contexte burkinabé. Chez les habitants du Plateau Mossi, Guiella, et al. (2000) trouvent que le travail effectué par les femmes et les enfants constitue la clé de voûte du système de production. D'autres études ont montré que les logiques lignagères et productives conduisent à une accumulation des femmes qui n'a pas pour seul avantage d'accéder à une force de travail d'usage immédiat (Michel lzard, 1985). Elles permettent également, par les enfants auxquels elles donnent naissance d'accumuler une force de travail d'usage différé dans l'agriculture et l'élevage. Certains chercheurs trouvent qu'une grande partie du temps de travail des enfants serait d'ailleurs consacrée à l'entretien et à la surveillance des animaux (Sawadogo et Larivière, 1993 ; Singh, 1988, (Cités par Guiella G. et Poirier J., 2000)). Dans un tel contexte, une modernisation du système de production pourrait avoir comme résultat une baise des besoins en main d'œuvre infantile et conduire ainsi à un niveau de fécondité désirée plus faible. Dans ce contexte, la disponibilité et l'utilisation des méthodes de contraceptions conduiraient à une fécondité plus faible car dans les sociétés modernes, la probabilité de conception est largement influencée par le niveau d'utilisation et d'efficacité de la contraception (KuateDefo 1998).

\section{Les facteurs de la contraception}

Plusieurs recherches se sont penchées sur l'analyse des facteurs de la contraception en Afrique (Guillaume et Pilon, 2000 ; Akoto et al. 1998 ; ANOH et al. 2004, Congo, 2005; Evina, 200I...). De la littérature, il ressort que deux grands groupes de facteurs influencent l'utilisation de la contraception. Les facteurs liés à l'offre contraceptive et ceux relatifs à la demande contraceptive. Les facteurs d'offre dépendent des politiques de planification familiale tandis que la demande est tributaire des besoins de la population. Ces facteurs agissent au niveau individuel, au niveau familial ou collectif (Guillaume et Pilon, 2000 : 15). Ainsi, la demande insatisfaite renvoie aux écarts existant entre les aspirations et les pratiques des individus en matière de fécondité (Andro, $A$ et Hertrich, $V$ p.724). Les facteurs qui influencent la demande contraceptive sont d'ordre démographique, socioéconomique et socioculturel.

Dans plusieurs pays étudiés, il ressort que le fait de vivre en ville favorise avant tout la pratique contraceptive moderne (Evina, A et Ngoy, K 200I), tandis qu'il constitue un facteur défavorable à la pratique contraceptive traditionnelle. Congo (2005) a montré que l'utilisation de la contraception moderne au Burkina Faso semble être réservée aux femmes résidant en milieu urbain. En travaillant sur le contexte du Cameroun, Evina (2005), trouve que la résidence de la femme en milieu urbain au moment de l'enquête, augmente sa propension à la pratique contraceptive. En effet, dans les villes, en plus de l'information sur la contraception, les populations ont un plus grand accès aux produits et services contraceptifs, notamment dans des formations sanitaires disposant de personnel qualifié ; ce qui favorise non seulement une plus grande prévalence contraceptive, mais aussi, l'utilisation des méthodes souhaitées par les femmes.

La scolarisation, par l'introduction des connaissances nouvelles ou par le degré d'acculturation qu'elle crée, constitue un facteur important qui favorise le recours à la planification familiale (Ngueyap, 1994). Cette relation se traduit par l'existence d'une volonté plus grande de planifier sa descendance chez les couples les plus instruits. L'éducation serait donc un facteur de modernisation qui apporte une meilleure connaissance des méthodes. Elle agit progressivement sur les normes et valeurs en matière de fécondité, qui constituent les déterminants cruciaux de la pratique contraceptive (Noumbissi et al. 1994, p/85). En plus de la connaissance, pourraient intervenir les moyens économiques pour se procurer les méthodes à utiliser. Pourtant, il y a certaines femmes qui connaissent la contraception moderne et ont les moyens de se procurer une méthode, mais n'y ont jamais eu recours (Noumbissi et al. 1994). Certains chercheurs trouvent que la pratique contraceptive varie avec le niveau d'ancrage des populations dans les pesanteurs socioculturelles. Dans ce sens, l'ethnie a souvent servi de variable proxy pour cerner l'effet des normes socioculturelles (Caldwell \& Caldwell 1993, Kritz \& Makinwa-Adebusoye 1999, Cité par Adjiwanou 2013) dans l'analyse des indicateurs de contraception et de fécondité. Quant à la religion, elle peut souvent constituer une barrière à l'acceptation de la contraception. Cependant, dans certaines études sur le contexte africain, il ressort que les pratiquants de la religion chrétienne sont nettement les plus favorables à la planification familiale (Ngueyap 1994). "Cette contradiction ne fait que traduire le décalage pouvant exister entre un 
discours institutionnel normatif et les représentations et pratiques individuelles »(Ngueyap 1994, p 175). Comme il ressort des lignes précédentes, plusieurs études ont exploré les questions liant les politiques de planification familiale et la fécondité en Afrique et au Burkina Faso. La plupart de ces études ont mis en exergue ou confirmé l'importance de la planification dans le processus de réduction de la fécondité. D'autres ont abordé l'influence de la contraception sur la fécondité à travers des analyses concernant une année donnée. Cependant, peu d'entre elles ont fait une analyse visant à identifier les sources de la baisse de la fécondité en relation avec l'évolution du contexte. C'est ce vide que cet article s'emploie à combler à travers l'utilisation de la méthode de la décomposition.

\section{CADRE THEORIQUE ET CONCEPTUEL}

Le cadre théorique et conceptuel a été adapté des travaux de Evina A et Ngoy K (200I). Ces auteurs admettent que les évolutions du cadre institutionnel (les politiques et programme de développement et les politiques de population) et les contextes socioéconomique et culturel influencent non seulement l'offre en matière de contraception mais aussi le désir d'enfants. En effet, les politiques de population et de planification peuvent agir sur la connaissance et l'utilisation de la contraception en facilitant l'accès aux services de planification familiale à toutes les couches de la population.

Les autres politiques de développement agissent aussi les facteurs socio-économiques et culturels tels que l'éducation et l'urbanisation. Ces facteurs ne sont pas des créneaux d'intervention des politiques de population mais leurs variations influencent les pratiques des populations en matière de planification familiale et de fécondité.

Les autres politiques de développement comme celles dans le domaine de l'éducation, de lutte contre la pauvreté, d'urbanisation pourraient être à l'origine d'un changement de structure des populations vis-àvis de ces caractéristiques. Par exemple, les études montrent que les femmes instruites ont des comportements plus favorables à une moindre fécondité. Ainsi, si leur proportion augmente on assistera à une baisse de la fécondité même en l'absence de politique de planification familiale. A l'opposé, si le niveau d'éducation restait inchangé, les politiques de planification familiale à travers les campagnes de sensibilisation et de promotion des méthodes contraceptives pourraient amener les femmes à connaître et à utiliser des méthodes contraceptives. Cela induirait également des perceptions et des pratiques reproductrices qui tendraient vers une hausse de la demande de produits contraceptifs dont la satisfaction conduirait à une moindre fécondité. Ainsi, le cadre conceptuel met en relation plusieurs variables qui sont à la fois influencées par les politiques de développement et les politiques de population. II s'agit de : la connaissance et l'utilisation des moyens de contraception, le nombre d'enfants désiré et le nombre d'enfants par femme.

A travers ce cadre conceptuel, nous cherchons à mesurer la contribution des politiques de planification familiale dans les changements en matière de contraception et de fécondité en cherchant à vérifier les hypothèses suivantes : (I) L'amélioration du niveau de connaissance et de pratique contraceptive est plus issue d'un effet de comportement que d'un effet de composition ; (2) La part de l'effet de comportement est plus grande dans les changements de la pratique contraceptive que dans ceux de la fécondité ; (3) La baisse de I'ISF est beaucoup plus liée à un changement des comportements de fécondité qu'à la hausse de la prévalence contraceptive moderne.

\section{DONNEES ET METHODES}

Cet article a utilisé plusieurs sources de données :

Les données quantitatives sont issues des Enquêtes démographiques et de santé (EDS) de 1998 et de 2010 du Burkina Faso. Ces deux enquêtes ont été retenues pour permettre d'apprécier l'évolution des indicateurs dans la décennie 2000 à 2009 avec la mise en œuvre de la PNP de 2000. L'enquête de 1998 a été réalisée avant la mise en œuvre de la PNP de 2000 tandis que celle de 2010 intervient à la fin. Les données des EDS de 1998 et de 2010 concernent respectivement 6445 femmes et 17087 femmes de 15-49 ans. Nous avons également utilisé les rapports d'analyse des RGPH de 2006 et de 1996 du Burkina Faso.

Les documents des Politiques nationales de population de 199| et 2000 et les plans d'action en matière de population (PAP) de 199I-1995 et de 2000-2005 ont été exploités. Ces documents ont été exploités à travers une grille de lecture qui permettait de renseigner les mesures prises, les activités réalisées, les résultats obtenus. Une lecture critique de ces documents a permis de mettre en exergue les limites et les perspectives en matière de planification familiale.

Les données qualitatives ont été collectées en 2012, dans le cadre de la rédaction d'un mémoire sur le thème "Politiques de population, réduction de la pauvreté et planification familiale au Burkina Faso ». Ces données ont été collectées à l'aide d'un guide d'entretien individuel semi-structuré. Les interviews ont concerné 12 personnes ressources, responsables de structures œuvrant dans le domaine de la 
population et du développement au Burkina Faso. II s'agit notamment des acteurs ayant travaillé à la Direction des Politiques de Population (ex. Conseil national de la Population), à l'Institut National de la Statistique et de la Démographie, à l'Association Burkinabé pour le Bien-être Familial etc. Une analyse du contenu a été faite du corpus issu de ces entretiens.

Les variables suivantes ont été utilisées :

ISF : Indice Synthétique de Fécondité : C'est le nombre moyen d'enfants nés vivants qu'aurait mis au monde une femme arrivée à la fin de sa vie féconde, si elle connaissait, à chaque âge, les taux de fécondité observés au cours d'une période de référence donnée ;

ISFD : c'est I'ISF Désiré qui est calculé de manière analogue à l'ISF en éliminant du numérateur les naissances considérées comme non désirées ;
La
connaissance
de
la

contraception moderne : c'est le fait de connaître ou non au moins une méthode contraceptive moderne ;

L'utilisation de la contraception moderne : c'est l'utilisation actuelle d'une méthode contraceptive moderne. Signalons que la variable adaptée ici est " le fait d'avoir déjà utilisé " mais elle n'est pas valide dans la base de données de l'EDS de 2010 ;

Le niveau d'instruction : niveau d'études formelles atteint par un individu : aucun niveau, primaire, secondaire ou plus ;

- Le milieu de résidence : lieu de vie suivant la qualification : urbain, rural.

Nous avons retenu les variables sur la connaissance et l'utilisation des méthodes contraceptives modernes car les méthodes traditionnelles sont faiblement utilisées et ne font pas l'objet de promotion par les politiques et les stratégies de planification familiale.

Dans les analyses, nous avons calculé la répartition des femmes selon le niveau d'instruction, le milieu de résidence et selon la pratique contraceptive en 1998 et en 2010. Nous avons également les taux de connaissance de la contraception moderne, la prévalence contraceptive moderne, l'ISFD et I'ISF selon les modalités du niveau d'instruction, du milieu de résidence et la pratique contraceptive en 1998 et en 20I0. Un indicateur d'évolution a été calculé en faisant la différence entre les chiffres de 1998 et 2010. Cet indicateur traduit la variation brute du taux entre les deux dates.

La méthode de la décomposition sert à mettre en exergue les contributions des différentes sources du changement social observé dans la variation des indicateurs au cours de la période de 1998 à 2010. Elle distingue deux sources principales de changement social à partir du changement global. Le changement global représente le changement total des indicateurs au niveau national. La décomposition permet d'analyser : (i) l'effet de composition qui est une statistique qui résulte d'un changement dans la distribution des femmes selon le niveau d'instruction ou le milieu de résidence et (ii) l'effet de comportement/performance qui indique un changement réel des indicateurs pour chacune des catégorie de femmes même si les distributions restaient constantes entre les deux dates.

La décomposition simple se fait suivant la formule :

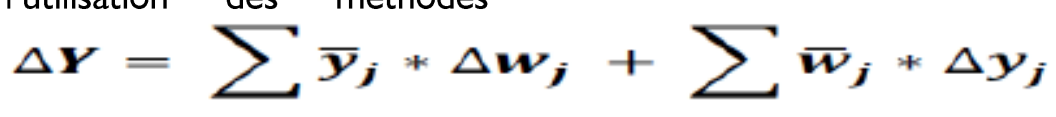

Effet total $=$ Effet de composition + Effet de comportement

Où $\Delta \boldsymbol{Y}$ est la variation de l'indicateur entre les deux dates et $\overline{\boldsymbol{y}}_{\boldsymbol{j}}$ la valeur moyenne de l'indicateur pour la catégorie $j$.

Dans cette étude, les politiques de population sont opérationnalisées à travers les actions de planification familiale. Comme expliqué dans le cadre conceptuel, les proportions de changement liées à l'effet de comportement sont attribuées aux résultats de la politique de planification familiale tandis que les proportions de changement liées à l'effet de composition sont interprétés comme les résultats des autres politiques de développement dont les rôles ont été, entre autres, l'amélioration du niveau d'instruction et du milieu de vie.

\section{Resultats}

Le tableau I présente l'évolution de quelques indicateurs sur la population du Burkina, notamment les indicateurs sur l'urbanisation et l'éducation de base. Nous pouvons noter, à partir des chiffres disponibles, que le taux d'urbanisation est passé de $15,5 \%$ en 1996 à $22,7 \%$ en 2006 . Quant au taux brut de scolarisation au primaire, il est passé de $40,8 \%$ en 1998 à $74,8 \%$ en 2010 .

Efforts de maîtrise de la fécondité au Burkina Faso L'analyse des sources documentaires sur les politiques de population montre que les efforts de régulation de la population à travers la planification familiale ont commencé en 1976, avec l'introduction dans les écoles de l'éducation en matière de 
population. En 1983, on a assisté à la création du par l'adoption en mai 1986 d'un Plan d'Action National en matière de planification familiale. La mise en œuvre de ce plan a nécessité différentes mesures législatives concernant essentiellement : l'abrogation de la loi de 1920 dans sa partie défavorable à la promotion des méthodes contraceptives (Tankoano FA 1991). Plusieurs autres textes qui s'inscrivent dans
Conseil national de la population (CONAPO), suivie le sens d'une transformation des perceptions et des comportements en matière de santé de la reproduction ont été adoptés. Par la suite, la Politique nationale de population de 1991 a été adoptée et mise en œuvre à travers son programme d'action en matière de population (PAP I) sur la période |99|-1995.

Tableau I : Evolution de quelques chiffres sur la population du Burkina Faso

\begin{tabular}{|c|c|c|c|c|c|}
\hline & 1985 & 1996 & 1998 & 2006 & 2010 \\
\hline Effectif de la population & 7964705 & 10312609 & - & 14017262 & 15730977 \\
\hline $\begin{array}{c}\text { Taux de croissance } \\
\text { intercensitaire }\end{array}$ & 2,7 & 2,4 & - & 3,1 & - \\
\hline Espérance de vie & 48,5 & 53,8 & - & 56,7 & \\
\hline Taux d'urbanisation & 12,7 & 15,5 & - & 22,7 & - \\
\hline $\begin{array}{l}\text { Taux brut de scolarisation } \\
\text { au primaire }\end{array}$ & & 38,42 & 40,8 & 67,0 & 74,8 \\
\hline
\end{tabular}

Sources : Analyse des résultats du RGP85 du Burkina Faso Enquête démographique (1991) et RGPH 1996, INSD, 2000, analyse des résultats du RGPH96 du Burkina Faso, volume l, Analyse des résultats du RGPH2006 du Burkina Faso, Thème : Etat et structure de la population, Projection de population, recensement général de la population et de l'habitation (2006), Tableau de bord social de l'INSD 2000, 2005 et 201 I

La politique nationale de population de 1991 a mis l'accent sur le droit fondamental des individus à décider de façon responsable de la taille de leur famille. Quant au plan d'action national en matière de planification familiale, elle visait le bien-être familial et social. Après les recommandations de la Conférence du Caire en 1994, la Politique nationale de population (PNP) a été révisé en 2000. Le nouveau but était l'atteinte de l'équilibre entre l'accroissement de la population et le développement socio-économique avec la mise en œuvre du deuxième plan d'action en matière de population (PAP II) qui couvrait la période 200 I-2005. Dans le cadre de la mise en œuvre de ces programmes d'action en matière de population, plusieurs documents et modules de formation ont été élaborés et utilisés pour la formation des animateurs et des intervenants dans le domaine de la planification familiale. Des formations à l'endroit des services sectoriels centraux et déconcentrés se sont déroulées. Elles visaient l'intégration des variables relatives à la population dans les politiques sectorielles. En plus, des activités de sensibilisation sur la planification familiale et contre la pratique de l'excision ont été menées. Le pays a été appuyé dans ces efforts par plusieurs ONG telles que l'ABBEF (créée en 1979, membre de l'IPPF depuis 1989) et le système de Nations Unies à travers l'UNFPA.

Connaissance et utilisation des méthodes contraceptives modernes

Les données présentées ici concernent les femmes en union de 15-49 ans. Le tableau 2 montre que le niveau de connaissance des méthodes contraceptives modernes a augmenté entre 1998 et 2010 en passant de $77,2 \%$ à $97,5 \%$ soit une augmentation de 20,3 points de pourcentage. Les femmes non instruites et celles du milieu rural qui étaient les moins informées en 1998 enregistrent les plus grandes évolutions en 2010.

Tableau 2 : Evolution du taux de connaissance des méthodes contraceptives modernes selon le niveau d'instruction et le milieu de résidence entre 1998 et 2010

\begin{tabular}{|c|c|c|c|c|c|c|c|c|}
\hline \multirow{3}{*}{ Caractéristiques } & \multicolumn{3}{|c|}{$\begin{array}{c}\text { Répartition des } \\
\text { femmes en union } \\
\text { de } 15-49 \text { ans (\%) }\end{array}$} & \multicolumn{3}{|c|}{$\begin{array}{l}\text { TCCM* femmes } \\
\text { en union de } \\
\text { I5-49 ans }\end{array}$} & \multirow{2}{*}{$\begin{array}{c}\text { Effet de } \\
\text { composition } \\
\text { (\%) }\end{array}$} & \multirow{2}{*}{$\begin{array}{c}\text { Effet de } \\
\text { comportemen } \\
(\%)\end{array}$} \\
\hline & 1998 & 2010 & Ecart & 1998 & 2010 & Ecart & & \\
\hline & \multicolumn{8}{|c|}{ Niveau d'instruction } \\
\hline Aucun niveau & 90,56 & 82,01 & $-8,55$ & 77,4 & 97,19 & 19,79 & \multirow{2}{*}{5,20} & \multirow{2}{*}{94,80} \\
\hline Primaire & 6,45 & 11,12 & 4,67 & 95,16 & 99,4 & 4,24 & & \\
\hline
\end{tabular}




\begin{tabular}{|ccccccccc|}
\hline Secondaire+ & 2,99 & 6,87 & 3,88 & 100 & 99,89 & $-0,11$ & \\
Urbain & 12,6 & 21,18 & 8,58 & 97,83 & 99,48 & 1,65 & \multirow{2}{*}{ Milieu de résidence } & \\
\cline { 2 - 9 } Rural & 87,4 & 78,82 & $-8,58$ & 76,54 & 97,13 & 20,59 & & 94,49 \\
\hline Burkina Faso & 100 & 100 & 0 & 77,2 & 97,5 & 20,3 & & \\
\hline
\end{tabular}

* : Taux de connaissance des méthodes contraceptives modernes; Sources de données : EDS du Burkina Faso de 1998 et 2010

Le taux d'utilisation des méthodes contraceptives modernes chez les femmes en union était de $15,0 \%$ en 2010 contre $4,8 \%$ en 1998 (Tableau 4). Depuis cette date, on a noté d'importantes évolutions dans les pratiques contraceptives chez les femmes vivant en milieu urbain et celles ayant un niveau d'instruction primaire. Malgré la réduction des écarts entre catégories de 1998 à 2010 , on note toujours d’importantes inégalités.

Tableau 3 : Evolution du taux d'utilisation de la contraception moderne selon le niveau d'instruction et le milieu de résidence entre 1998 et 2010

\begin{tabular}{|c|c|c|c|c|c|c|c|c|}
\hline \multirow{3}{*}{ Caractéristiques } & \multicolumn{3}{|c|}{$\begin{array}{l}\text { Répartition des } \\
\text { femmes en union } \\
\text { de } 15-49 \text { ans (\%) }\end{array}$} & \multicolumn{3}{|c|}{$\begin{array}{l}\text { TPCM* femmes } \\
\text { en union de } \\
\text { 15-49 ans }\end{array}$} & \multirow{2}{*}{$\begin{array}{c}\text { Effet de } \\
\text { composition } \\
(\%)\end{array}$} & \multirow{2}{*}{$\begin{array}{c}\text { Effet de } \\
\text { comportement } \\
(\%) \\
\end{array}$} \\
\hline & 1998 & 2010 & Ecart & 1998 & 2010 & Ecart & & \\
\hline & \multicolumn{8}{|c|}{ Niveau d'instruction } \\
\hline Aucun niveau & 90,56 & 82,01 & $-8,55$ & 3,19 & 11,19 & 8 & \multirow{4}{*}{18,12} & \multirow{4}{*}{81,88} \\
\hline Primaire & 6,45 & 11,12 & 4,67 & 13,07 & 25,2 & 12,13 & & \\
\hline Secondaire+ & 2,99 & 6,87 & 3,88 & 36,61 & 44,23 & 7,62 & & \\
\hline & \multirow{2}{*}{\multicolumn{7}{|c|}{ Milieu de résidence }} & \\
\hline Urbain & 12,6 & & & & & & & \multirow{2}{*}{84,22} \\
\hline Rural & 87,4 & 78,82 & $-8,58$ & 2,63 & 10,78 & 8,15 & 15,78 & \\
\hline Burkina Faso & 100 & 100 & 0 & 4,83 & 15,02 & 10,19 & & \\
\hline
\end{tabular}

* : Taux de prévalence contraceptive moderne ; Sources de données : EDS du Burkina Faso de 1998 et 2010

Le graphique I montre que les changements observés dans les niveaux de connaissance et dans la pratique contraceptive sont plus liés à un effet de comportement (près de $95 \%$ pour la connaissance et
$82 \%$ pour la pratique). Ce qui tend à montrer une légère augmentation de la contribution de l'effet de composition quand on passe de la connaissance à la pratique de la contraception

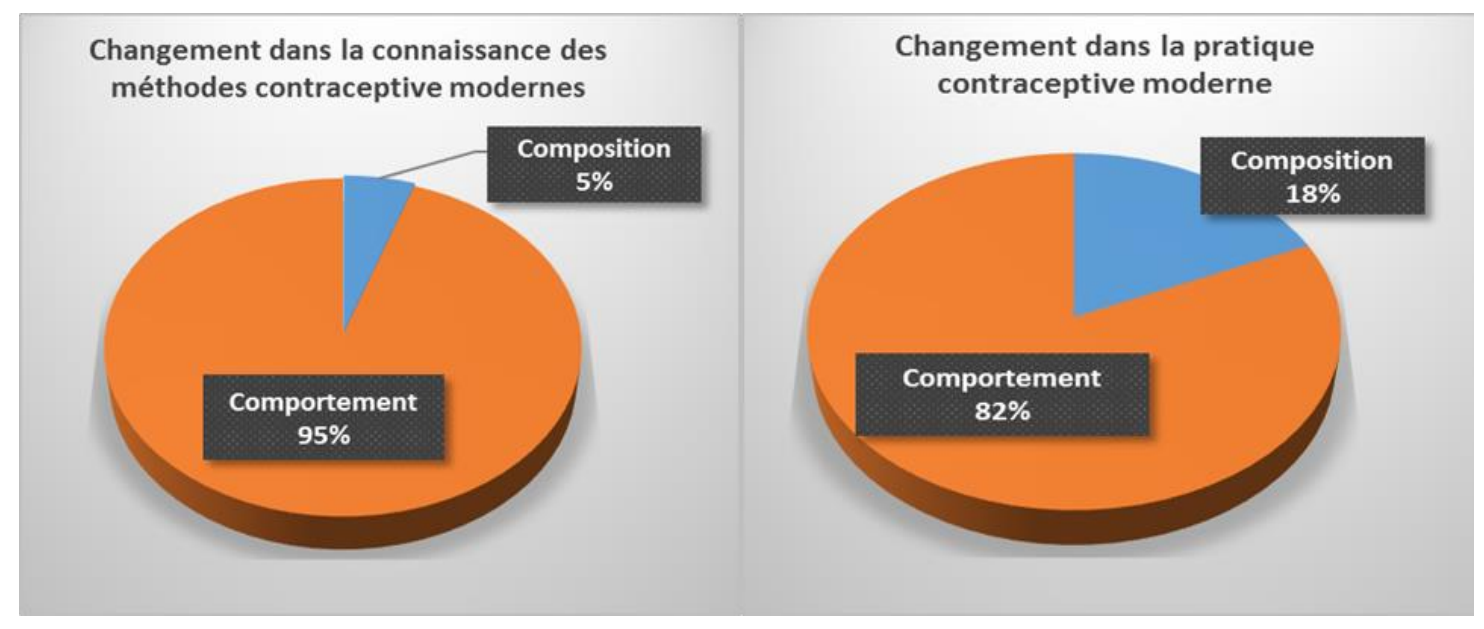

Graphique I : Parts de contribution des sources de changement dans la connaissance et la pratique contraceptive moderne 
Préférences et pratiques en matière de la fécondité

D'une façon générale, on assiste à une réduction du nombre d'enfants désiré qui passe de 6 enfants par femme à 5,2 enfants par femme entre 1998 et 2010 .

Selon les catégories, les plus forts pourcentages de baisse de l'ISFD s'observent chez les femmes de la modalité " aucun niveau " et chez les femmes du milieu rural (Tableau 4).

Tableau 4 : Evolution de l'indice synthétique de fécondité désirée par femme selon le niveau d'instruction et le milieu de résidence de 1998 à 2010

\begin{tabular}{|c|c|c|c|c|c|c|c|c|}
\hline \multirow{3}{*}{ Caractéristiques } & \multicolumn{3}{|c|}{$\begin{array}{l}\text { Répartition des } \\
\text { femmes de } \\
\text { 15-49 ans (\%) }\end{array}$} & \multicolumn{3}{|c|}{$\begin{array}{l}\text { ISFD* toutes } \\
\text { les femmes } \\
\text { de } 15-49 \text { ans }\end{array}$} & \multirow{2}{*}{$\begin{array}{c}\text { Effet de } \\
\text { composition } \\
(\%)\end{array}$} & \multirow{2}{*}{$\begin{array}{c}\text { Effet de } \\
\text { comportement } \\
(\%)\end{array}$} \\
\hline & 1998 & 2010 & Ecart & 1998 & 2010 & Ecart & & \\
\hline & \multicolumn{8}{|c|}{ Niveau d'instruction } \\
\hline Aucun niveau & 85,8 & 73,9 & $-11,9$ & 6,3 & 5,8 & $-0,5$ & \multirow{4}{*}{46,76} & \multirow{4}{*}{53,24} \\
\hline Primaire & 8,4 & 13,6 & 5,2 & 4,2 & 4,2 & 0 & & \\
\hline Secondaire+ & 5,8 & 12,4 & 6,6 & 2,5 & 2,8 & 0,3 & & \\
\hline & \multicolumn{6}{|c|}{ Milieu de résidence } & & \\
\hline Urbain & 16,9 & 27,1 & 10,2 & 3,4 & 3,3 & $-0,1$ & \multirow{2}{*}{37,24} & \multirow{2}{*}{62,76} \\
\hline Rural & 83,1 & 72,9 & $-10,2$ & 6,5 & 5,9 & $-0,6$ & & \\
\hline Burkina Faso & 100 & 100 & 0 & 6,00 & 5,20 & $-0,80$ & & \\
\hline
\end{tabular}

* : Nombre moyen d'enfants désiré par femme de $15-49$ ans calculé pour les naissances des trois dernières années précédant l'enquête ;

Sources de données : EDS du Burkina Faso de 1998 et 2010

En ce qui concerne la fécondité, on note une baisse progressive de I'ISF qui passe de 6,7 enfants par femme en 1998 à 6 enfants par femme en 2010. L'analyse selon les caractéristiques des femmes montre que les baisses les plus importantes dans la fécondité sont observées chez les groupes ayant une plus grande fécondité en 1998. II s'agit notamment des femmes avec " aucun niveau " d'instruction, celles de niveau primaire et celles vivant en milieu rural.

Tableau 5 : Evolution du nombre moyen d'enfants par femme selon le niveau d'instruction et le milieu de résidence entre 1998 et 2010

\begin{tabular}{|c|c|c|c|c|c|c|c|c|}
\hline \multirow[b]{2}{*}{ Caractéristiques } & \multicolumn{3}{|c|}{$\begin{array}{l}\text { Répartition des } \\
\text { femmes de } \\
\text { 15-49 ans (\%) }\end{array}$} & \multicolumn{3}{|c|}{$\begin{array}{l}\text { ISF* toutes les } \\
\text { femmes de } \\
\text { I5-49 ans }\end{array}$} & \multirow{2}{*}{$\begin{array}{c}\text { Effet de } \\
\text { composition } \\
(\%)\end{array}$} & \multirow{2}{*}{$\begin{array}{c}\text { Effet de } \\
\text { comportement } \\
(\%)\end{array}$} \\
\hline & 1998 & 2010 & Ecart & 1998 & 2010 & Ecart & & \\
\hline & \multicolumn{8}{|c|}{$\overline{\text { Niveau d'instruction }}$} \\
\hline Aucun niveau & 85,8 & 73,9 & $-11,9$ & 7,1 & 6,6 & $-0,5$ & \multirow{4}{*}{44,48} & \multirow{4}{*}{55,52} \\
\hline Primaire & 8,4 & 13,6 & 5,2 & 5,4 & 4,9 & $-0,5$ & & \\
\hline Secondaire + & 5,8 & 12,4 & 6,6 & 2,9 & 3,1 & 0,2 & & \\
\hline & \multicolumn{6}{|c|}{ Milieu de résidence } & & \\
\hline Urbain & 16,9 & 27,1 & 10,2 & 4,1 & 3,9 & $-0,2$ & \multirow{2}{*}{37,41} & \multirow{2}{*}{62,59} \\
\hline Rural & 83,1 & 72,9 & $-10,2$ & 7,3 & 6,7 & $-0,6$ & & \\
\hline Burkina Faso & 100 & 100 & 0 & 6,70 & 6,00 & $-0,70$ & & \\
\hline
\end{tabular}

* : Nombre moyen d'enfants par femme de $15-49$ ans, calculé pour les naissances des trois dernières années précédant l'enquête ; Sources de données : EDS du Burkina Faso de 1998 et 2010

La décomposition de la variation de l'ISF fait ressortir une plus grande proportion liée au changement de comportement (56\%), qui traduit une baisse de l'ISF au sein des femmes contre un effet de composition 
(44\%), lié à une amélioration du niveau d'instruction. et de comportement sont assez similaires pour l'ISF et l'ISFD.

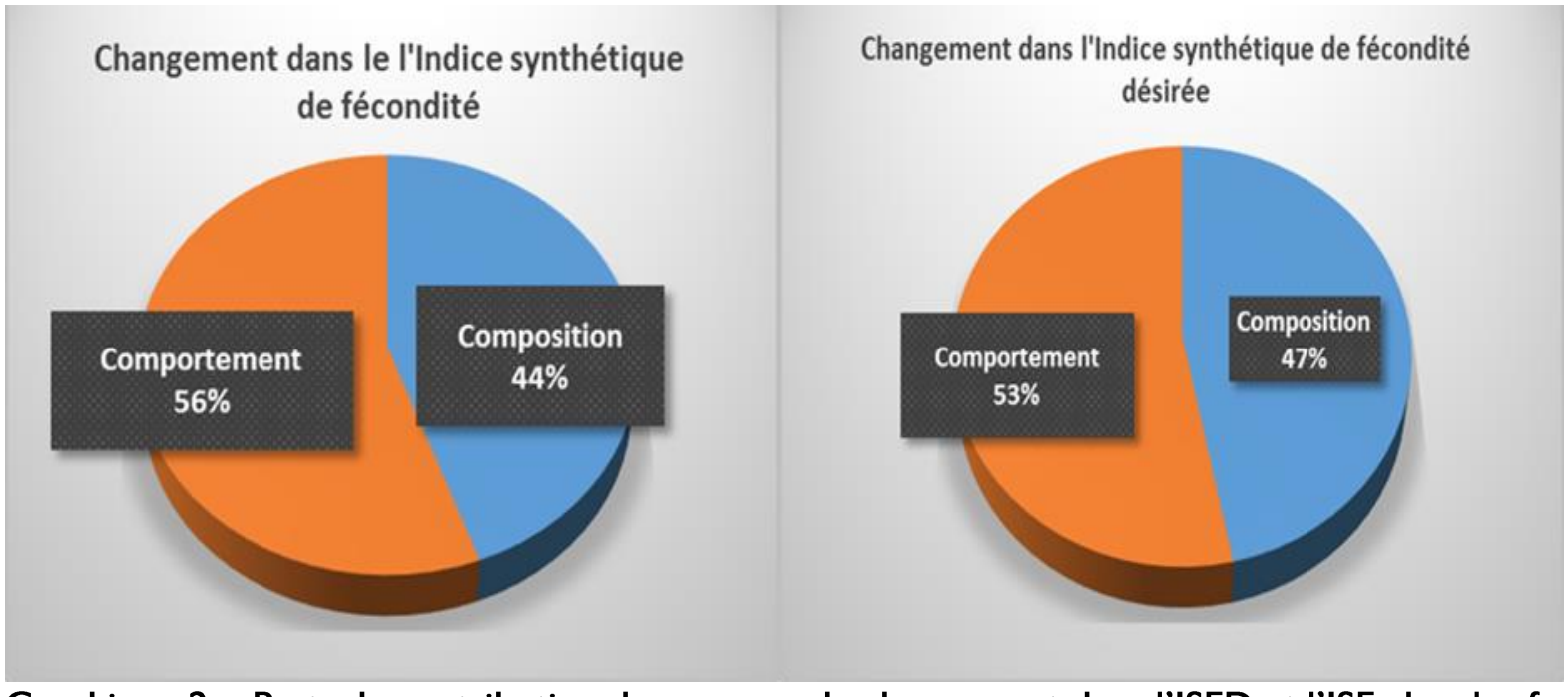

Graphique 2 : Parts de contribution des sources de changement dans l'ISFD et l'ISF chez les femmes de $15-49$ ans

Impact de la pratique contraceptive moderne sur la baisse de la fécondité

Le tableau présente la variation de l'ISF des femmes selon qu'elles utilisent ou non la contraception moderne entre 1998 et 2010 . Dans cette partie, nous avons utilisé I'ISF des 12 derniers mois pour rapprocher la période de référence de celle de l'utilisation actuelle de la contraception. Nous
Les parts de contribution des effets de composition

Tableau 6 : Evolution du nombre moyen d'enfants par femme selon l'utilisation de la contraception moderne entre 1998 et 2010

\begin{tabular}{|c|c|c|c|c|c|c|c|c|}
\hline \multirow{3}{*}{$\begin{array}{c}\text { Caractéristiques } \\
\text { Utilise }\end{array}$} & \multicolumn{3}{|c|}{$\begin{array}{c}\text { Utilisation de la } \\
\text { contraception moderne : } \\
\text { toutes les femmes de I5-49 } \\
\text { ans (\%) }\end{array}$} & \multicolumn{3}{|c|}{$\begin{array}{l}\text { ISF* : toutes les } \\
\text { femmes de } \\
\text { I5-49 ans }\end{array}$} & \multirow{2}{*}{$\begin{array}{c}\text { Effet de } \\
\text { composition } \\
(\%)\end{array}$} & \multirow{2}{*}{$\begin{array}{c}\text { Effet de } \\
\text { comportement } \\
(\%) \\
\end{array}$} \\
\hline & 1998 & 2010 & Ecart & 1998 & 2010 & Ecart & & \\
\hline & 5,83 & 14,26 & 8,43 & 3,47 & 2,97 & $-0,5$ & & \\
\hline N'utilise pas & 94,17 & 85,74 & $-8,43$ & 7,24 & 6,7 & $-0,54$ & 37,10 & 62,90 \\
\hline Burkina Faso & 100 & 100 & 0 & 7,02 & 6,17 & $-0,85$ & & \\
\hline
\end{tabular}

* : Nombre moyen d'enfants par femme de $15-49$ ans, calculé pour les naissances des 12 derniers mois précédant l'enquête ; Sources de données : EDS du Burkina Faso de 1998 et 2010

\section{Discussion}

Les résultats montrent que les indicateurs de la planication familiale ont connu des évolutions notables entre 1998 et 2010. Les changements observés sont liés à des effets de composition et de comportement mais dans des proportions inégales.

Sources du changement dans la connaissance et dans la pratique contraceptive moderne

http://aps.journals.ac.za assistons à une baisse de l'ISF dans les deux groupes de femmes. Le niveau de baisse de l'ISF est un peu plus important chez les femmes qui n'utilisent pas la contraception moderne. Ces changements sont liés principalement à un effet de comportement des femmes $(62,9 \%)$ contre $37,10 \%$ des changements qui sont liés à l'augmentation de la proportion des femmes qui utilisent la contraception moderne. 
sont liés à une amélioration du niveau de connaissance et d'utilisation des méthodes contraceptives par les femmes. Ces effets pourraient donc être attribués aux performances des campagnes d'information et de sensibilisation menées entre les deux dates dans le cadre des politiques de planification familiale. Pendant ce temps, les effets de composition qui illustrent les contributions issues de l'éducation et de l'urbanisation restent très faibles, environ $5 \%$ pour la connaissance et $18 \%$ pour l'utilisation (Tableau 2). La faiblesse de l'effet de composition montre que les variations dans la répartition des femmes selon le milieu de résidence et le niveau d'instruction n'ont pas eu d'effets susceptibles d'influencer la connaissance de la contraception et la pratique contraceptive moderne. Comme on l'a vu dans la partie précédente, une amélioration du niveau d'instruction et une croissance de la proportion urbaine de la population sont des facteurs d'un plus large accès des femmes aux canaux d'information, aux produits et aux services sur la planification familiale.

Une comparaison entre les sources du changement dans la connaissance et les sources du changement dans la pratique contraceptive montre que l'effet de comportement est plus grand dans les variations de la connaissance que dans les variations de la pratique contraceptive (Graphique I). Cela est la preuve d'un moindre impact des politiques de planification familiale sur l'amélioration du niveau de la pratique contraceptive comparativement au niveau de connaissance. D'où la preuve de la persistance voir l'augmentation du fossé entre la connaissance et la pratique de la contraception moderne. Cette situation pourrait s'expliquer soit par la volonté des couples de ne pas utiliser les méthodes connues pour des raisons diverses. Selon certains chercheurs, ces raisons peuvent être réunies en trois grands groupes : la perception des femmes de ne pas être à risque de grossesse, la non disponibilité de produits et de services de planification familiale adéquats ainsi que la peur des effets secondaires de la contraception, l'opposition à la planification familiale de la part de la femme ou de son partenaire (Sedgh, et al, 2007).

Les changements observés en matière de connaissance et de pratique de la contraception entre 1998 et 2010 tiennent leurs sources non seulement de l'effet de composition, mais aussi d'un effet de comportement. L'effet de comportement étant la plus importante, (plus de $80 \%$ de contribution (Tableau 2 et 3 )), nous pouvons conclure que les politiques de planification familiale sont les principales sources de ces changements. En effet, on pourrait dire que ces politiques, à travers les activités de sensibilisation sont parvenues à accroitre sensiblement le niveau de connaissance et d'utilisation des méthodes contraceptives modernes.

Apport de la planification familiale sur la baisse de I'ISFD et de I'ISF

Les tableaux 4 et 5 montrent que, avec l'analyse selon le niveau d'instruction, l'effet de comportement est plus important dans la baisse de I'ISFD et l'ISF, respectivement $53,24 \%$ et $55,52 \%$. Ces contributions liées aux changements de comportement sont assez proches de $50 \%$. Ce qui montre que les effets de composition sont aussi importants que ceux relatifs au comportement. On pourrait aussi noter qu'une plus grande augmentation de la proportion des femmes instruites aurait permis d'avoir une baisse plus grande du désir d'enfants. En effet, avoir un niveau d'instruction élevé, implique d'offrir de meilleures conditions sanitaires et éducatives à sa descendance notamment en milieu urbain. C'est ce que trouve Noumbissi A (1994 : p323) : «en se généralisant, l'urbanisation, l'instruction et les institutions non familiales entraînent des changements de comportement en matière de procréation et ce à travers les facteurs sociaux : relâchement de la division sexuelle des rôles qui pesait sur la femme, évolution du système familial, diminution de la valeur affective et sociale des enfants; et les facteurs ". Du fait de la scolarisation, les femmes de niveau d'instruction élevé ont tendance à rentrer tardivement dans la vie féconde, ce qui a pour conséquence de réduire la durée d'exposition à la fécondité. D'où la baisse de la fécondité chez ces femmes comparativement aux femmes de niveau d'instruction faible. Cela explique en partie les différences de nombre moyen d'enfant entre les femmes selon le niveau d'instruction. Plus haut, nous avons vu que les femmes du milieu urbain sont mieux informées et utilisent plus la planification familiale que leurs consœurs du milieu rural. Cela pourrait aussi être dû aux conditions de vie modernes dans ce milieu caractérisé par un plus grand accès à l'information.

L'impact de la contraception sur la fécondité est aussi prouvé dans la mesure où selon le tableau 6 , les femmes qui utilisent la contraception moderne ont en moyenne moins d'enfants que celles qui ne l'utilisent pas. Cependant, l'impact de la hausse du niveau de contraception moderne sur la fécondité est assez mitigé car nous remarquons qu'une plus grande baisse de I'ISF chez les femmes qui n'utilise pas la contraception moderne. La décomposition de I'ISF selon l'utilisation de la contraception montre que seulement $37,1 \%$ de la baisse de l'ISF est liée à la hausse de la prévalence contraceptive. Cela tend à confirmer un changement de comportement de ces femmes tendant vers une réduction de leur fécondité http://aps.journals.ac.za 
sans nécessairement avoir recours à la contraception moderne. Ce constat pourrait suggérer que ces femmes utilisent des méthodes traditionnelles ou naturelles pour éviter ou retarder les grossesses. Toutefois, les les desirs et les stratégies d'espacement ou d'arrêt dépendent de plusieurs autres facteurs non abordés ici. D'autres analyses poussées sur la contribution des facteurs tels que le désir d'enfant et la contraception traditionnelle pourraient permettre d'élucider la question. Par ailleurs, l'utilisation actuelle de la contraception pour évaluer l'impact sur la fécondité présente une limite en ce sens que la fécondité qui est mesurée concerne une période passée plus longue que la période de référence de l'utilisation de la contraception.

Comme il a été montré à travers cette étude, la baisse de la fécondité est favorisée par la hausse du niveau de connaissance et d'utilisation de la contraception moderne. Cependant, comparées à d'autres variables socioéconomiques, ces variables n'ont souvent que de faibles contributions. La hausse de la prévalence contraceptive a notamment une contribution de moins de $40 \%$. Ainsi, en plus des politiques de planification familiale, l'Etat doit mettre l'accent sur les autres politiques telles que l'éducation, la santé et l'amélioration des conditions de vie.

\section{Limites des politiques de population sur la réduction de la fécondité}

A travers l'analyse des sources documentaires, notamment des rapports de synthèse des activités des PAP, on peut noter que les textes adoptés au Burkina Faso dans le cadre de la réglementation du développement et de la croissance démographique ont canalisé la mise en œuvre des actions de Planification familiale. Ces actions ont été développées sous l'influence du Cadre stratégique de lutte contre la pauvreté (CSLP). L'analyse des données qualitatives fait ressortir que le caractère simultané des deux politiques (PNP et du CSLP) n'a pas permis qu'elles soient efficaces. En effet, selon plusieurs acteurs interviewés, la mise en œuvre des PNP a été contrariée par l'avènement des Programmes d'Ajustement Structurel (PAS) dans les années 1990. A partir des années 2000 , on note l'adoption du CSLP comme cadre unique de référence pour les politiques publiques dans lequel les questions démographiques ont été très faiblement prises en compte. En plus, la persistance d'une grande proportion de la population dans le secteur agro-pastoral utilisant des méthodes de production précaires est une des conséquences de l'échec des politiques de développement. Sur le plan sanitaire, l'éloignement des centres de santé des populations et la forte médicalisation des services de planification http://aps.journals.ac.za familiale n'ont pas permis une bonne promotion de l'utilisation des méthodes contraceptives.

Les données quantitatives permettent de noter que les politiques de planification familiale ont eu une plus grande contribution dans les changements de la connaissance et de la pratique contraceptive (plus de $80 \%$ ). Cependant, en ce qui concerne les changements au niveau de la fécondité, la contribution de la pratique contraceptive est assez mitigée (moins de 40\%) selon le tableau 6. Cela constitue une des limites de l'action des politiques de planification familiale sur la baisse de la fécondité dans la mesure où elles agissent sur la fécondité à travers l'utilisation des méthodes contraceptives modernes. La question qui se pose est : pourquoi l'amélioration du niveau d'utilisation n'est pas proportionnellement suivie d'une baisse de la fécondité ? La réponse se situe à plusieurs niveaux dont le fait qu'une majorité des femmes utilisent dans le but d'un espacement des naissances (Congo, 2005 : 10). Ce constat avait été fait par Van de Walle (1988:160, cité par Congo, (2005: 10)) qui disait que : «si les stratégies d'espacement des naissances sont généralement évidentes, celles d'arrêt sont rares. Dans les campagnes (surtout) comme en milieu urbain, une progéniture nombreuse demeure toujours un signe de prospérité, de main-d'œuvre, d'assurance retraite et de garantie pour ses vieux jours ". Ainsi, la persistance d'une fécondité encore élevée malgré une plus grande utilisation de la contraception serait la conséquence de leur utilisation à des fins d'espacement plutôt qu'à des fins de limitation des naissances. Les données issues des entretiens et des sources documentaires suggèrent que la baisse de la demande d'enfant pourrait intervenir suite à une modernisation des secteurs de production qui ont beaucoup recours au travail des enfants. C'est ce que pense Dakuyo et al., (2010 : iii), "si la demande d'enfants reste élevée en milieu rural, c'est parce que la modernisation de l'agriculture n'a pas été réussie et aussi parce que la scolarisation n'a pas atteint ses ambitions d'universalité »

Défis et perspectives en matière de réduction de la fécondité

En somme, on peut retenir que les politiques de population, à travers la planification familiale ont des impacts notables sur les changements dans la contraception et les préférences de fécondité. Cependant, elles ont été limitées dans leur impact sur la fécondité. Ainsi, la poursuite des efforts de réduction de la fécondité présente plusieurs défis que nous rangeons dans deux domaines.

Dans le domaine de la planification familiale, le Burkina Faso devra s'inspirer des expériences réussies dans d'autres pays. En effet, nous notons 
qu'il existe plusieurs types d'expériences dans le domaine de la maîtrise de la fécondité. Cependant, les acteurs intervenant dans le domaine des politiques de population ont été unanimes à citer les cas de la Tunisie et du Ghana comme des exemples réussis de maîtrise de la croissance démographique. En plus, il faut noter l'amélioration de la qualité des services de planification familiale. Des études réalisées ont montré qu'une meilleure qualité des services de planification familiale est un facteur favorisant une plus grande demande. La satisfaction des clientes est associée à l'utilisation efficace et continue d'une méthode, et aux rapports " de bouche à oreille". Inversement, une interaction client-prestataire médiocre est associée à la cessation de l'usage d'une méthode ou à son échec. A titre d'exemple, des recherches réalisées en Egypte ont révélé que les consultations axées sur les clientes (au lieu de celles axées sur le médecin) étaient associées à un niveau trois fois plus élevé de satisfaction des clientes et de continuité dans l'utilisation de la méthode choisie. Par ailleurs, le Burkina Faso pourra s'inspirer de l'expérience du Ghana qui a mis l'accent sur la mobilisation communautaire autour de la question de planification familiale. En effet, dans ce pays où la planification familiale s'est propagée rapidement avec un fort impact sur la fécondité, on a pu remarquer une forte implication des communautés dans le processus de diffusion. Au Burkina Faso, les services de planification familiale sont restés l'apanage des formations sanitaires qui semblent avoir perdu leur vocation de promotion sociale. Pourtant, s'il est vrai que l'utilisation de certaines méthodes requiert le concours d'un personnel de santé, d'autres, par contre, peuvent être utilisées sans trop d'intermédiaires.

Dans le domaine des autres politiques de développement, un accent doit être mis sur l'éducation des filles. En effet, plusieurs pays du Maghreb ont été cités comme ayant des exemples réussis dans la baisse de la fécondité. II est ressorti que ces pays ont mis l'accent sur un plus grand accès à l'éducation, suivi d'une plus grande prévalence des emplois salariés notamment des femmes qui, de ce fait, ne disposeraient pas d'assez de temps pour s'occuper d'une fécondité élevée. L'instruction des filles semble être le facteur le plus commun à la plupart des cas de réduction de la fécondité. Dans une analyse de la transition démographique dans les pays du Maghreb, il ressort que : "si les programmes de limitation des naissances plus classiques n'ont pas vraiment fait la preuve d'une grande efficacité, d'autres politiques, ne visant pas a priori ce but, en ont eu bien davantage. Ce sont celles qui ont permis de rendre quasi universelle l'instruction de base, notamment celle des filles, celles qui ont ouvert le marché du travail aux femmes, celles qui ont amélioré leur statut familial, social, économique et culturel. Autant de facteurs assez largement communs aux trois pays du Maghreb pour expliquer la similitude de leurs évolutions» (Vallin, 2012 :3). Certains répondants ont mis l'accent sur les conditions favorables à un changement de perception à travers les changements introduits dans les structures économiques. II s'agit notamment de la scolarisation obligatoire, de la mécanisation de l'agriculture, de l'expansion de la sécurité sociale à travers une plus grande salarisation des emplois.

Ces différentes transformations qui ne sont pas des facteurs directs de la fécondité ont pour but d'augmenter le coût de l'enfant en termes de prise en charge et de réduire sa contribution aux activités productrices des parents. Dans ce processus, on assistera à une croissance de la demande contraceptive. Ainsi, la disponibilité des services et produits de planification familiale pourra effectivement permettre aux populations d'avoir la capacité d'espacer ou de limiter volontairement les naissances.

\section{Conclusion}

Cet article s'est penché sur les interrelations entre la politique de population, la planification familiale et leurs contributions à la baisse de la fécondité. A l'issue de cette analyse, il ressort que le niveau de la pratique de la contraception moderne accuse toujours un retard sur le niveau de connaissance qui fait croire à l'existence d'un fossé entre la connaissance des méthodes et leur utilisation. On a aussi démontré que les politiques de population et de planification familiale ont beaucoup plus contribué à l'amélioration des connaissances des méthodes contraceptives qu'à leur utilisation. D'autre part, un désir d'enfants qui est encore élevé semble mitiger l'impact de la hausse de la prévalence contraceptive moderne sur la baisse de la fécondité. Ce qui montre l'importance des autres politiques de développement dont les politiques éducatives et d'urbanisation dans une baisse soutenue de la fécondité.

A l'issue de l'article, nous formulons les recommandations suivantes :

la poursuite des politiques de planification familiale, notamment le plan de relance de planification familiale, en mettant l'accent sur la formation des prestataires ;

l'inclusion dans les programmes de planification familiale des stratégies de promotion des 
- méthodes contraceptives basées sur le système décentralisé et orienté vers les femmes et des filles non instruites ;

- la mise en œuvre de programmes visant la modernisation du secteur agricole, la salarisation des emplois et l'expansion de la sécurité sociale de sorte à induire une transformation profonde dans les structures socioéconomiques.

\section{Bibliographie}

Andro, A et Hertrich, V 200I, 'La demande contraceptive au Sahel : les attentes des hommes se rapprochent-elles de celles de leurs épouses?' Population (French Edition), Vol. 56, No. 5 (Sep. Oct., 200I), pp. 72I-77I

Anoh, A, Fassassi R et Vimard P 2004, 'Politique de population et planification familiale en Côte d'ivoire', in Gautier A. (éd) Les politiques de planification familiale : cinq expériences nationales, |95-23|.

Burkina Faso 2000, Politique Nationale de la Population du Burkina Faso. Révision $\mathrm{N}^{\circ} \mathrm{I}$, CONAPO, MEF.

Burkina Faso 2013, Plan national de relance de la Planification Familiale 20I3-20I5

Dakuyo, LM, Koné, $M$ et Wetta, C 20I0, Réflexion sur les défis de la croissance démographique au Burkina Faso, VISION «BURKINA 2025 ».

Direction Générale de l'Economie et de la Planification 201I, Rapport sur les finances publiques Burkina Faso 2010.

Evina A et Ngoy, K 200 I, "L'utilisation des méthodes contraceptives en Afrique : de l'espacement à la limitation des naissances" in Gendreau, $F$ et Poupard, $M$ Les transitions démographiques dans les pays du sud. Actualité Scientifique. 253-268.

Institut National de la Statistique et de la Démographie (INSD) et ICF International 20I2, Enquête Démographique et de Santé et à Indicateurs Multiples du Burkina Faso 2010. Calverton, Maryland, USA : INSD et ICF International.

Institut National de la Statistique et de la Démographie 1995, Analyse des résultats de l'enquête démographique 1991. 2ème édition.

Institut National de la Statistique et de la Démographie 2009, Thème 2 : Etat et structure de la population, analyse des résultats définitifs du recensement général de la population et de l'habitation (RGPH) de 2006.

Institut National de la Statistique et de la Démographie 2012, Tableau de bord social du Burkina Faso $201 \mathrm{l}$.

Ministère de la Santé, 2006, Plan d'accélération de Réduction de la Mortalité Maternelle et Néonatale au Burkina Faso.

Ministère de la Santé, 2014, tableau de bord 2014 des indicateurs de santé, Burkina Faso.

Murphy, E et Steele, C 2000, Interactions clientsprestataires en matière de planification

familiale : lignes directrices émanant des travaux de recherche et de l'expérience des programmes. Document MAQ, vol. I, N $\mathrm{N}^{\circ},-2000$, http://www.maqweb.org/maqdoc/french/vol2.htm [5/25/20I। I2:22:05 PM]

Noumbissi, A 1994, 'Fécondité et développement : de quelques théories', in Koffi, N, Guillaume, A, Vimard, P et Zanou, B (ed), Maîtrise de la croissance démographique et développement en Afrique, ORSTOM 199I, 321-337.

Organisation Mondiale de la Santé 2015, Tendances de la mortalité maternelle 1990-2015: Estimations de l'OMS, I'UNICEF, I'UNFPA, le Groupe de la Banque mondiale et la Division de la population des Nations Unies, Résumé d'orientation

Sedgh, G, Hussain, R, Bankole, A and Singh, S 2007, Women with an Unmet Need for Contraception in Developing Countries and Their Reasons for Not Using a Method, Occasional Report No. 37, June 2007.

Tankoano, AF 199I, 'L'expérience de planification familiale au Burkina Faso' in Koffi, N, Guillaume, A, Vimard, P et Zanou, B (ed), Maîtrise de la croissance démographique et développement en Afrique, ORSTOM 199I, 233-250

Vallin, J 2012, 'Faut-il une politique de population ?' Population \& Sociétés, INED, $N^{\circ}$ 489, Mai 2012, I-4.

Van de Walle, E 1988, 'Les pratiques traditionnelles et modernes des couples en matière d'espacement ou d'arrêt de la fécondité' in Tabutin D. (dir), Population et sociétés en Afrique au sud du Sahara. L'Harmattan, Paris : | 4|- 66. 